\title{
Transitional Flow Dynamics Behind a Micro-Ramp
}

\author{
J. Casacuberta ${ }^{1} \cdot$ K. J. Groot ${ }^{1} \cdot$ Q. Ye $\mathrm{e}^{1} \cdot$ S. Hickel ${ }^{1}$
}

Received: 29 March 2019 / Accepted: 1 October 2019 / Published online: 19 December 2019

(C) The Author(s) 2019

\begin{abstract}
Micro-ramps are popular passive flow control devices which can delay flow separation by re-energising the lower portion of the boundary layer. We compute the laminar base flow, the instantaneous transitional flow, and the mean flow around a micro-ramp immersed in a quasi-incompressible boundary layer at supercritical roughness Reynolds number. Results of our Direct Numerical Simulations (DNS) are compared with results of BiLocal stability analysis on the DNS base flow and independent tomographic Particle Image Velocimetry (tomo-PIV) experiments. We analyse relevant flow structures developing in the micro-ramp wake and assess their role in the micro-ramp functionality, i.e., in increasing the near-wall momentum. The main flow feature of the base flow is a pair of streamwise counter-rotating vortices induced by the micro-ramp, the so-called primary vortex pair. In the instantaneous transitional flow, the primary vortex pair breaks up into large-scale hairpin vortices, which arise due to linear varicose instability of the base flow, and unsteady secondary vortices develop. Instantaneous vortical structures obtained by DNS and experiments are in good agreement. Matching linear disturbance growth rates from DNS and linear stability analysis are obtained until eight micro-ramp heights downstream of the micro-ramp. For the setup considered in this article, we show that the working principle of the micro-ramp is different from that of classical vortex generators; we find that transitional perturbations are more efficient in increasing the near-wall momentum in the mean flow than the laminar primary vortices in the base flow.
\end{abstract}

Keywords DNS · Micro-ramp · Laminar-turbulent transition · Roughness · SFD · Hairpins · BiLocal stability · Tomo-PIV

\section{Introduction}

Laminar-turbulent transition in boundary layer flows is, despite its high relevance in many aerodynamic applications, still far from being fully understood and it remains an active

J. Casacuberta

J.CasacubertaPuig@tudelft.nl

S. Hickel

S.Hickel@tudelft.nl

1 Faculty of Aerospace Engineering, TU Delft, Kluyverweg 1, 2629HS Delft, The Netherlands 
research topic within the aerodynamics community. Transition is affected by various factors, among which wall imperfections may play a central role. Interest in the effect of roughness in the transition process comes, first of all, from the necessity to understand the interaction between laminar fluid flow and realistic surfaces [1-3]. Secondly, surface roughness can either accelerate or delay the transition process $[4,5]$, which is of interest in flow control applications. In this work, we focus on the dynamics of boundary layer transition in the incompressible flow regime when it is promoted by an isolated three-dimensional roughness element. Transition predictions for this class of problems generally rely on the roughness Reynolds number, which is defined as

$$
R e_{h h}=\frac{u_{h} h}{v_{\infty}}
$$

where $h$ is the height of the roughness element, $v_{\infty}$ is the free-stream viscosity, and $u_{h}$ is the streamwise velocity of the unperturbed boundary layer at the element's height [1].

At subcritical $R e_{h h}$ conditions, i.e., if the critical $R e_{h h}$ value is not exceeded, the flow remains laminar. Above the critical $R e_{h h}$ value, at supercritical $R e_{h h}$ conditions, the roughness element starts to affect the transition process and increasing $R e_{h h}$ moves the transition location upstream towards the roughness location. Despite being widely used, correlations of $R e_{h h}$ are based on empirical work and are valid only for simple flow configurations. As Schneider [6] points out, there is still no general mechanisms-based model that defines the conditions under which a roughness element causes transition.

Experimental work performed at low-speed conditions has nonetheless provided reasonable understanding about the flow mechanisms underlying the roughness-induced transition process [7]. Isolated roughness elements distort the laminar boundary layer and create a wake of increased streamwise vorticity and shear. Vortex filaments redistribute momentum, which induces positive and negative streaks. The resulting detached shear layer is susceptible to Kelvin-Helmholtz $(\mathrm{K}-\mathrm{H})$ instability. This conception is typically reinforced by the observation of vortex shedding [3]. The roughness-induced shedding of hairpin vortices was first reported by Acarlar \& Smith [8] and Klebanoff et al. [9] for the wake of a single hemispherical roughness. Similar flow topologies have been found also for other roughness geometries [10,11].

For the analysis presented in this article, we consider a micro-ramp vortex generator as the roughness shape used to trigger laminar-turbulent transition. A micro-ramp -which is essentially a forward wedge, see Fig. 1- is a passive flow control device that can reduce flow separation by increasing near-wall momentum. Its advantages are a low drag penalty and structural robustness. The micro-ramp wake topology has been widely investigated in the past decade, predominantly in supersonic and turbulent flow conditions, where they can be used to reduce flow separation caused by shock-wave/boundary-layer interaction (SWBLI) [12-14], for example. Fluid lifted at the leading edge of the micro-ramp descends from the slant edges and induces a pair of counter-rotating vortices, the primary vortex pair, which enhances momentum redistribution within the boundary layer. The current consensus attributes a reduction of the shape factor of the boundary layer to entrainment of highmomentum fluid towards the wall under the action of the primary vortices $[12,13,15,16]$. Some authors have nonetheless recently questioned the micro-ramp working principle. For example, Wang et al. [17] claim that the primary vortices are not capable of entraining a sufficient amount of high-momentum fluid from the free-stream to the wall downstream 
Fig. 1 Micro-ramp geometry

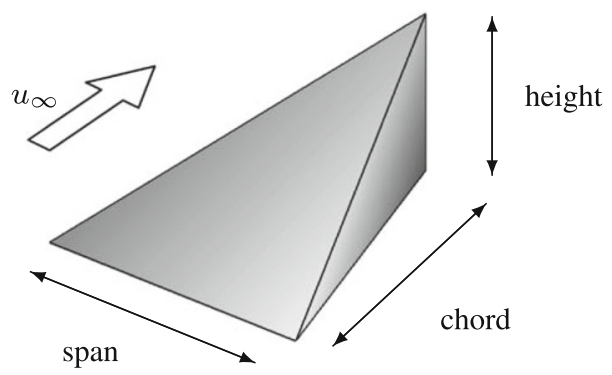

of the micro-ramp. Instead, it is proposed that the mechanism which makes the boundary layer fuller relies on the exchange of high- and low-momentum fluid of the boundary layer directly at the micro-ramp location. Li and Liu [18] attribute a reduction of the separation bubble not to increased wall shear due to the primary vortices' motion, but to the fact that arc-shaped vortices induced at a detached shear layer weaken the shock. They suggest that due to this effect, the micro-ramp working principle is different from that of traditional vortex generators. Bo et al. [19] hypothesise that the basic micro-ramp flow organisation is, at first, the consequence of the motion of the primary vortices and, thereafter, maintained by hairpin-vortex dynamics. Blinde et al. [20] report that there is no evidence of the activity of the primary vortices in the instantaneous flow field sufficiently downstream from the micro-ramp. There is consensus on the fact that the micro-ramp is capable of re-energising the lower part of the boundary layer and make it less prone to separation; however, what mechanisms cause this effect is disputable.

In this article, we present and discuss the results of DNS of a low-speed $M_{\infty}=0.2$ micro-ramp flow at supercritical $R e_{h h}$ conditions. We analyse relevant flow structures and transitional mechanisms present in the micro-ramp wake, and assess the potential of this device to accelerate the transition process. Furthermore, we inquire into the relative contribution of the (laminar) primary vortices on the one hand, and the transitional perturbations on the other hand to the increase of the near-wall momentum. We believe that the current analysis provides a better understanding of the momentum-transport mechanisms that may be partially convoluted in more complex flow environments. The novelty of our approach lies in that, to characterise the effect of transitional perturbation development in the micro-ramp functionality, we additionally compute the laminar steady flow, the unstable base flow, and perform a BiLocal stability analysis of the unstable micro-ramp wake.

Upon comparing the base flow and the fully developed instantaneous transitional flow field sufficiently far downstream of the micro-ramp, differences can be exclusively attributed to the effects of finite-amplitude disturbances. Close to the micro-ramp, exponential growth of perturbations in the linear amplification stage is captured. Therefore, the growth of small-amplitude disturbances measured from the results of DNS is compared with the results of BiLocal stability analysis performed on the unstable micro-ramp base flow.

This article is structured as follows: in Section 2, the flow problem and the details of the DNS setup are presented. In Section 3, we discuss the results of the numerical simulations: first, the topology of the base flow and the transitional flow and, secondly, the generation of momentum excess in the micro-ramp wake. We conclude the article with Section 4. 


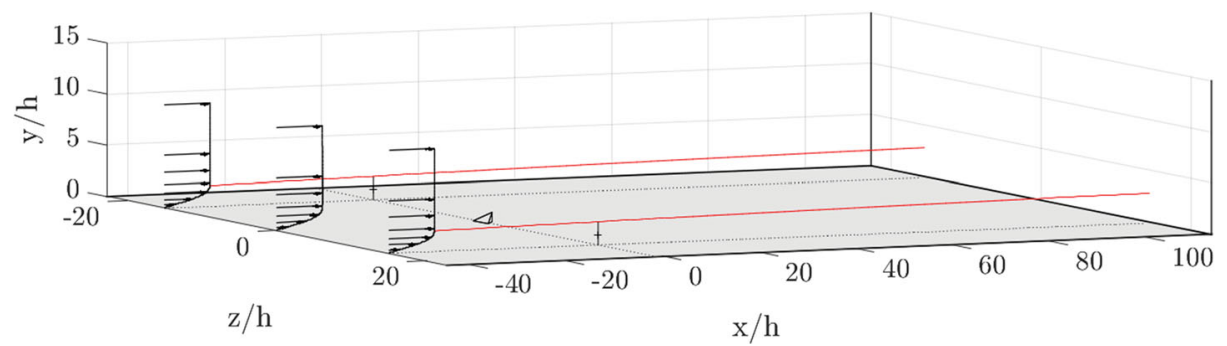

Fig. 2 Sketch of the micro-ramp flow problem with the micro-ramp geometry, inlet boundary layer profiles (computed at $x / h=-45$ and plotted at $z / h=0$ and $z / h= \pm 15$ ), height of the undisturbed boundary layer (red solid line) and its projection onto the wall (black dashed line), ticks at $x / h=-1.25$ indicating the micro-ramp height. Streamwise and spanwise domain size matches the computational box

\section{Methodology}

\subsection{Direct numerical simulations}

We solve the compressible Navier-Stokes equations to determine the flow field around a micro-ramp mounted on a flat plate and immersed in a laminar $M_{\infty}=0.2$ boundary layer. Figure 2 depicts a sketch of the flow problem. A two-dimensional boundary layer-obtained as the solution of the self-similar compressible boundary layer equations- is imposed at the inlet, develops along the surface, and encounters the micro-ramp. For our analysis, we use a micro-ramp with height $h$, span $2 h$ and chord $2.25 h$, which is one of the most used geometries in the literature $[11,12,14]$.

The DNS results are compared with the results of tomographic Particle Image Velocimetry (tomo-PIV) experiments carried out by Ye et al. [11]. Special attention was devoted in matching the flow conditions between DNS and the experiments. Table 1 summarises and compares relevant boundary-layer parameters between these two approaches. The parameter $R e_{h}$ is defined as

$$
R e_{h}=\frac{u_{\infty} h}{v_{\infty}},
$$

with $u_{\infty}$ being the free-stream velocity. It ought to be emphasised that in Ref. [11], $u_{h}$ and hence $R e_{h h}$ are computed based on flow properties at $x / h=-1.25$, thus slightly upstream of the micro-ramp tip. This location corresponds to where the micro-ramp was bolted to the flat plate in the experiments. For the sake of consistency, we follow this convention. The unperturbed boundary layer thickness at the micro-ramp bolt location is $\delta_{99}$, un $=h / 0.44$. At the inflow boundary, it equals $h / 0.52$.

The DNS of the micro-ramp flow are performed with the finite-volume immersedboundary method INCA, see Refs. [21, 22], for example. We use a computational box of dimensions $-45 \leq x / h \leq 114$ in the streamwise direction, $0 \leq y / h \leq 23$ in the wallnormal direction, and $-23 \leq z / h \leq 23$ in the spanwise direction. The micro-ramp tip is located at $(0, h, 0)$. The domain size is chosen such that boundaries are sufficiently far from

Table 1 Comparison of boundary layer parameters between PIV experiments [11] and the current work

\begin{tabular}{lllll}
\hline Case & $R e_{h}$ & $R e_{h h}$ & $h / \delta_{99, \text { un }}(x / h=-1.25)$ & $u_{h} / u_{\infty}$ \\
\hline PIV & 700 & 468 & 0.44 & 0.67 \\
DNS & 700 & 463 & 0.44 & 0.67 \\
\hline
\end{tabular}


the micro-ramp and we are conservative enough to capture all relevant transitional flow structures and mechanisms. Special concerns are the spanwise spreading and the remarkably large upwards propagation of the micro-ramp wake, and the streamwise coordinate at which the boundary layer starts to display turbulent behaviour [11]. The computational domain is divided in 430 blocks with Adaptive Mesh Refinement (AMR) and contains $5.66 \times 10^{6}$ hexahedral cells. The structured grid is highly refined around the micro-ramp and near the wall, guaranteeing $y^{+}<1$. The current grid resolution is sufficient to resolve the transitional flow downstream of the micro-ramp. We will demonstrate in Section 3.2 that the perturbation growth rates measured in the DNS match with those predicted by the linear stability analysis in the region close to the micro-ramp.

The compressible Navier-Stokes equations are marched in time with a third-order explicit Runge-Kutta (RK) method. The inviscid flux is reconstructed with a 5th-order Weighted Essentially Non-Oscillatory (WENO) [23] scheme with HLLC flux function [24]. Inflow and free-stream boundary conditions are imposed through Riemann invariants. The flat plate and micro-ramp are modelled as adiabatic no-slip walls. The Selective Frequency Damping (SFD) technique $[25,26]$ is used to compute the unstable steady-state base flow. Our choice for the SFD control parameters, $\chi=0.96$ and $\Delta=1.86$, is based on the stability results presented in Ref. [33] and the specifications on how to choose the SFD parameters discussed in Ref. [26]. Hereafter, the subscript "s" is used to denote steady laminar base flow quantities computed with SFD, while the time-averaged flow variables (mean flow) are expressed with “〈 $\rangle "$

\subsection{BiLocal stability analysis}

The literature $[19,20]$ indicates that unsteady perturbations play an important role in maintaining the high-speed streaks in the micro-ramp wake under supercritical $R e_{h h}$ conditions. For this reason, the perturbations' existential origin is investigated by analysing the stability of the base flow to infinitesimal perturbations. Such perturbations are governed by the linearised Navier-Stokes equations in general. Specific features of the investigated flow allow imposing further simplifications that reduce the complexity of the analysis.

First of all, the reverse-flow region behind the micro-ramp is very small and limited to $x / h<1$. For $x / h>1$, the base flow develops slowly in the streamwise direction. Therefore, it is reasonable to neglect the impact of streamwise gradients of the base flow on the perturbation dynamics. Second, as will be pointed out in Section 3.1, the stability mechanism of interest is convective, it is not an absolute nor global mechanism [27, 28]. Lastly, the Mach number is very small, $M_{\infty}=0.2$. In light of these features, it is justified to use the incompressible formulation of the BiLocal stability framework [29] (this is also referred to as the spanwise BiGlobal framework), which is set up as follows.

In assuming that the streamwise gradients of the flow can be neglected, the linearised Navier-Stokes equations have constant coefficients in $x$. In turn, that allows specifying the following perturbation ansatz:

$$
q^{\prime}(x, y, z, t)=\tilde{q}(y, z) \mathrm{e}^{\mathrm{i}(\alpha x-\omega t)}+c . c .
$$

where $q^{\prime}$ denotes an infinitesimal perturbation quantity, $\tilde{q}$ is a (complex) perturbation amplitude, $\omega$ the angular frequency, $\alpha$ the streamwise wavenumber and c.c. denotes "complex conjugate." This ansatz is used specifically for the velocity components in the streamwise $x$-, wall-normal $y$ - and spanwise $z$-direction: $u, v$ and $w$, respectively, and the pressure $p$. 
Substituting Ansatz (3) into the incompressible linearised Navier-Stokes equations yields

$$
\begin{array}{r}
\frac{\tilde{D} \tilde{u}}{\tilde{D} t}+\tilde{v} \frac{\partial u_{s}}{\partial y}+\tilde{w} \frac{\partial u_{s}}{\partial z}=-\mathrm{i} \alpha \tilde{p}+\frac{1}{R e}\left(-\alpha^{2}+\frac{\partial^{2}}{\partial y^{2}}+\frac{\partial^{2}}{\partial z^{2}}\right) \tilde{u}, \\
\frac{\tilde{D} \tilde{v}}{\tilde{D} t}+\tilde{v} \frac{\partial v_{s}}{\partial y}+\tilde{w} \frac{\partial v_{s}}{\partial z}=-\frac{\partial \tilde{p}}{\partial y}+\frac{1}{R e}\left(-\alpha^{2}+\frac{\partial^{2}}{\partial y^{2}}+\frac{\partial^{2}}{\partial z^{2}}\right) \tilde{v}, \\
\frac{\tilde{D} \tilde{w}}{\tilde{D} t}+\tilde{v} \frac{\partial w_{s}}{\partial y}+\tilde{w} \frac{\partial w_{s}}{\partial z}=-\frac{\partial \tilde{p}}{\partial z}+\frac{1}{R e}\left(-\alpha^{2}+\frac{\partial^{2}}{\partial y^{2}}+\frac{\partial^{2}}{\partial z^{2}}\right) \tilde{w}, \\
\mathrm{i} \alpha \tilde{u}+\frac{\partial \tilde{v}}{\partial y}+\frac{\partial \tilde{w}}{\partial z}=0,
\end{array}
$$

where

$$
\frac{\tilde{D}}{\tilde{D} t}=-\mathrm{i} \omega+\mathrm{i} \alpha u_{s}+v_{s} \frac{\partial}{\partial y}+w_{s} \frac{\partial}{\partial z} .
$$

Perturbation amplitudes $\{\tilde{u}, \tilde{v}, \tilde{w}, \tilde{p}\}$ are sought that decay far away from the shear layers of interest. This justifies truncating the considered $z y$-plane for the stability problem to: $(z, y) / h \in[-5.5,5.5] \times[0,13.4]$. Dirichlet conditions are used for the velocity amplitudes at all truncation boundaries. Neumann conditions are imposed on the pressure at the boundaries in the spanwise direction, while a Dirichlet condition is imposed at the top boundary, which resolves the additive non-uniqueness of the pressure field. At the wall, the no-slip condition is imposed for all velocity amplitudes and the $y$-momentum equation is used as a compatibility condition for the pressure amplitude. The results are found to be independent of the domain lengths and the chosen boundary conditions.

As mentioned, we are interested in perturbations that grow in the streamwise direction. Therefore a (real) frequency is imposed, implying zero temporal amplification. Given the boundary conditions, the base flow evaluated at a $z y$-plane at a downstream $x$-station of the micro-ramp and the frequency $\omega$, System (2.2) forms a quadratic eigenvalue problem, where $\alpha$ is the eigenvalue and $\tilde{u}, \tilde{v}, \tilde{w}$ and $\tilde{p}$ are eigenfunctions. The quadratic problem is cast into a linear eigenvalue problem through the companion matrix approach [30]. The problem is discretised using Chebyshev collocation [31] in both the $z$ - and $y$-directions. A BiQuadratic mapping is used [34], placing one-third of the collocation nodes in-between specified coordinate locations in the $y$-direction. Using 60 nodes in the $z$ - and $y$-directions yielded $\alpha$-eigenvalues converged up to $O\left(10^{-5}\right)$ absolute errors in units of $\mathrm{rad} / h$. In the streamwise direction, a stepsize $\Delta x=0.25 h$ was used.

The Arnoldi method is used to solve the eigenvalue problem [32]. The eigenvalue $\alpha$ is generally complex. Through Ansatz (3), $-\alpha_{i}$ represents the streamwise exponential growth rate of a perturbation at a considered $x$-station, where $i$ denotes the imaginary part. To compare against the DNS results, this growth rate is integrated to yield the $N$-factor:

$$
N(x)=-\int^{x} \alpha_{i}(\bar{x}) \mathrm{d} \bar{x},
$$

where the bar denotes a dummy integration variable. Several unstable modes are recovered, of which the most amplified perturbation is found to have a varicose symmetry with respect to the center-plane, see [33].

To reconstruct a three-dimensional representation of the $q^{\prime}$ perturbation variables (see Fig. 6), the $\tilde{q}$ eigenfunctions at different $x$-stations are stitched together with the following steps. First, the arbitrary phase of the eigenfunctions was aligned in the $x$-direction. 
This was done by rescaling the whole eigenvector such that the real part of the pressure amplitude function attains its absolute maximum, i.e., $\max _{z, y} \tilde{p}_{r}=\max _{z, y}|\tilde{p}|$, where the subscript $r$ denotes the real part. The pressure eigenfunction was chosen, because it has a very well-defined shape for the solution of interest. Second, the maximum amplitude of the $|\tilde{u}|$-eigenfunction, which has the largest overall amplitude, was set to unity in the $x$ direction. These steps are allowed because a complex multiple of an eigenvector is again an eigenvector, where an eigenvector is the vector of eigenfunctions, like: $\Xi=[\tilde{u}, \tilde{v}, \tilde{w}, \tilde{p}]^{T}$. Third, the (varying) streamwise wavenumber and the relative amplitude dictated by the $N$ factor were imposed by multiplying the eigenfunctions with $\exp \left(N(x)+\mathrm{i} \alpha_{r} x\right)$ and, fourth, the real part was taken.

Note that contributions from the eigenfunctions $\tilde{q}(y, z)$ are neglected in the definition of the $N$-factor. This is consistent with the assumption that they are locally constant in $x$, as encoded in Ansatz (3). Despite this argument and the unification of their size with the aforementioned steps, the eigenfunctions do still vary slightly from one $x$-station to the other. Therefore, the contribution of the eigenfunctions was quantified to ensure it did not affect the results. This was done by integrating the absolute value of each eigenfunction over the entire $z y$-plane and checking the evolution of the integral in $x$. The resulting differences in the $N$-factors for all variables was very small $(\Delta N<0.5)$ and varied very little with $x$, therefore these contributions are deemed negligible and excluded from the present results.

\subsection{Tomographic PIV}

The experiments [11] were carried out in the open jet low-speed wind tunnel at the Aerodynamics Laboratories of TU Delft. In the tomographic PIV setup, the flow was seeded with water-glycol droplets of approximately $1 \mu \mathrm{m}$ diameter. The measurement region was illuminated with a Quantel CFR PIV-200 Nd:YAG laser $(200 \mathrm{~mJ} /$ pulse, $532 \mathrm{~nm}$ wavelength, 9 ns pulse duration). Particle images are captured by four LaVision Imager Pro LX interline CCD cameras $(4872 \times 3248$ pixels, $7.4 \mu \mathrm{m} /$ pixel $)$ arranged along an arc. Each camera is equipped with an objective of $105 \mathrm{~mm}$ focal length, which were tilted to comply with the Scheimpflug condition. The snapshots are acquired at a frequency of $1.5 \mathrm{~Hz}$. The measurement volume is $145 \times 6 \times 45 \mathrm{~mm}^{3}(72.5 h \times 3 h \times 22.5 h)$, resulting in a digital image resolution of $33.6 \mathrm{pixel} / \mathrm{mm}$. In the streamwise direction, the domain extends from $2.5 \mathrm{~mm}$ to $147.5 \mathrm{~mm}$ behind the micro-ramp tip, covering the evolution from the laminar flow to a fully turbulent boundary layer. Three ramp heights are covered in the wall-normal direction.

\section{Results}

\subsection{Analysis overview}

The primary vortex pair emanating from the slant edges of the micro-ramp is reported in the literature as the main feature in micro-ramp flows [11-14]. Upon computation of the micro-ramp's base flow, we can isolate the structure of the primary vortices and analyse their momentum transport in absence of perturbations. Figure 3a depicts iso-surfaces of the streamwise vorticity $\left(\omega_{x}\right)$ in the base flow; the primary vortices are identified as streamwisedeveloping elongated symmetric structures aside the centre plane. Figure 4a portrays the wall shear in the base flow. The imprint of the primary vortices is evident; since they increase the near-wall momentum, symmetric regions of enhanced wall shear arise near the centre 

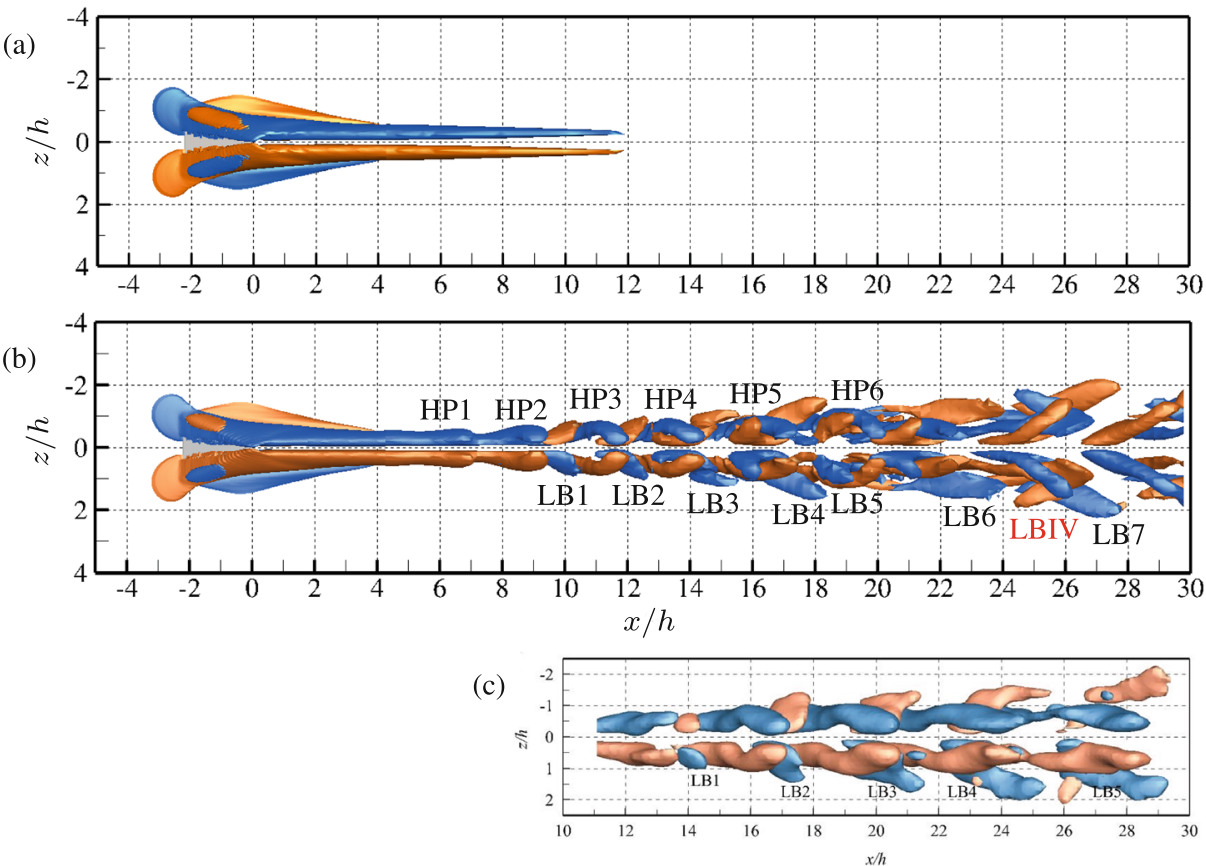

Fig. 3 Instantaneous streamwise vorticity from the (a) DNS base flow, (b) DNS transitional flow, (c) tomoPIV [11]. Isosurfaces of $\omega_{x} h / u_{\infty}= \pm 0.25(\mathbf{a}, \mathbf{b})$

plane. When moving downstream, the wall shear exhibits a rapid decay. Figure 4a thus suggests that the streamwise vortex filaments in the base flow are insufficient to maintain the near-wall flow energisation over a longer downstream distance.

(a)

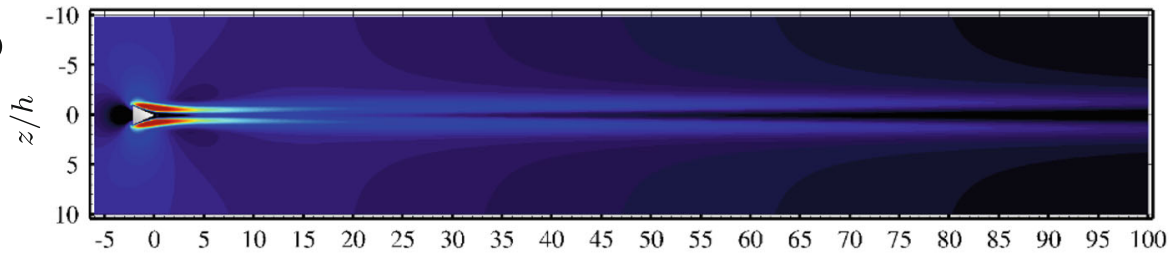

(b)

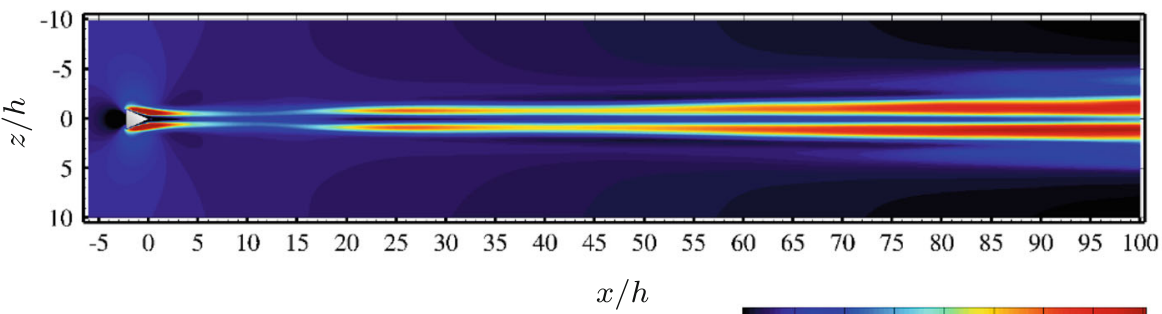

$s_{\mathrm{S}} h / u_{\infty},\langle s\rangle h / u_{\infty}: 0.400 .550 .700 .851 .001 .151 .301 .451 .60$

Fig. 4 Wall shear in the (a) base flow and (b) mean flow 
Based on their measurements, Ye et al. [11] indicate that the critical $R e_{h h}$ of the current micro-ramp setup is expected to lie in the range 320 to 460 . Our DNS corresponds to the supercritical case with $R e_{h h}=463$, see Table 1. Accordingly, we observe that perturbations start growing exponentially when SFD is switched off. We do not find a temporally growing localised structure around the micro-ramp, which would indicate a global or absolute instability mechanism. Instead, we find a convective mechanism from the moment that the SFD model is switched off onwards. As mentioned before, this justifies the use of the BiLocal stability framework, which is restricted to representing convective instability mechanisms in the way it is currently applied. Disturbance growth eventually leads to a saturated nonlinear transitional flow and new flow features appear, see Fig. 3b. Figure 4b depicts wall shear for the mean flow. By referring to the mean flow, we mean the time-averaged flow including the effect of non-linear perturbations. At a first glance, we observe major differences between the base-flow and mean-flow wall shear downstream of the micro-ramp. From $x / h=10$ onwards, the mean-flow wall shear is significantly higher. Moreover, the wake of the micro-ramp in the mean flow expands in the spanwise direction. Figure 4 hence highlights the importance of the perturbation development on improving the performance of the micro-ramp.

In the forthcoming discussion, we first describe and analyse the base flow and the instantaneous flow organisations of the micro-ramp wake (Section 3.2). Secondly, we inquire into the generation of momentum excess to explain the aforementioned differences between the base and the mean near-wall flow behaviour (Sections 3.3 and 3.4).

\subsection{Organisation of the base flow and the transitional flow}

The primary vortices are counter-rotating and induce upwash at the centre plane and a lateral downwash. The mutual vortex interaction causes them to rapidly lift off the surface. In the base flow, the primary vortex pair is found to remain within the boundary layer at all streamwise stations. Iso-surfaces of streamwise vorticity characterising the structure of the primary vortices (considering $\omega_{x, \mathrm{~s}} h / u_{\infty}= \pm 0.04$ ) never exceed $\delta_{99}$, un vertically. Even though the intensity of the vortices decays with $x / h$, as illustrated in Fig. 3a, they persist far downstream of the micro-ramp. For increasing $x / h$, they furthermore move slightly away from the centre plane. Very weak steady secondary vortices are captured as well, developing in between the primary vortex pair and the surface. However, we observe that these do not significantly contribute to the momentum redistribution at any streamwise position of the domain.

A different instantaneous vortical activity is observed in the transitional flow field; see Fig. 3b. Around and in the direct downstream vicinity of the micro-ramp, iso-surfaces of streamwise vorticity represent the primary vortex pair. These iso-surfaces show a steady behaviour and a spatial development similar to that observed in the base flow configuration. For increasing $x / h$, these structures gradually start to oscillate under the influence of unsteady perturbations. Sufficiently downstream of the micro-ramp, the primary vortex pair breaks up into a train of hairpin vortices, hereafter denoted by "HP" and, eventually, we find no more remnants of the primary vortex pair. Similar observations were made by Blinde et al. [20] considering a high-speed case. After the primary vortices break up, the legs of the hairpin vortices appear as instantaneous isosurfaces of streamwise vorticity of alternating sign (with respect to the centre plane) in Fig. 3 b.

The hairpin vortices develop due to an instability of the base flow. Stability analysis applied to the DNS base flow reveals that the wake of the micro-ramp supports two convectively unstable instabilities. The most unstable mode is of K-H type and follows a symmetric 
spatial distribution. Thus, it is referred to as a varicose mode. The second, less unstable mode shows a sinuous (anti-symmetric) spatial distribution. The varicose instability is the mechanism generating the hairpin vortices. Figure 6 compares the streamwise-velocity perturbation field obtained from instantaneous snapshots from DNS and tomo-PIV experiments with the stability eigenfunction of the most unstable mode. We have labelled the perturbation structures for which we find a topological match with Roman numerals in Fig. 6.

To quantify the perturbation growth, we compute

$$
\epsilon=\frac{1}{u_{\infty}^{2} h^{2}} \iint\left\langle u^{\prime \prime} u^{\prime \prime}\right\rangle \mathrm{d} y \mathrm{~d} z
$$

the integrated streamwise velocity perturbation energy; $u^{\prime \prime}$ expresses an instantaneous finiteamplitude fluctuation of streamwise velocity measured with respect to the mean flow. The streamwise evolution of $\epsilon$ is presented in Fig. 5 in $N$-factor form, that is, expressed as $\ln \left(\epsilon / \epsilon_{\mathrm{ref}}\right) / 2$, where $\epsilon_{\text {ref }}$ corresponds to the value of $\epsilon$ at $x / h=-2$ and the division by 2 is required because (7) measures a kinetic energy amplitude, while the $N$-factor represents the velocity amplitude. Figure 5 depicts the $N$-factor curve obtained from the results of the stability analysis as well.

The presented $N$-factor curve corresponding to the stability analysis is scaled such that it coincides with the amplitude measurement from the DNS at $x / h=6$. The $N$-factor curve represents a single frequency, $\omega h / u_{\infty}=1.4$, that was found to be the most unstable frequency for the range $x / h \in[0,24]$, up to a frequency difference of $\Delta \omega=0.1 u_{\infty} / h$. Therefore, it is also representative of the entire envelope of $N$-factor curves for different frequencies. This implies that the perturbations could be modelled as a wave train (being composed out of a single frequency) instead of a sequence of wave packets (created with multiple or a band of frequencies) up to a reasonable accuracy. The perturbations do manifest themselves as wave packets in the DNS, they do not form a perfect, non-modulating wave train. However, the length of the wave packets is large. Therefore this effect can be

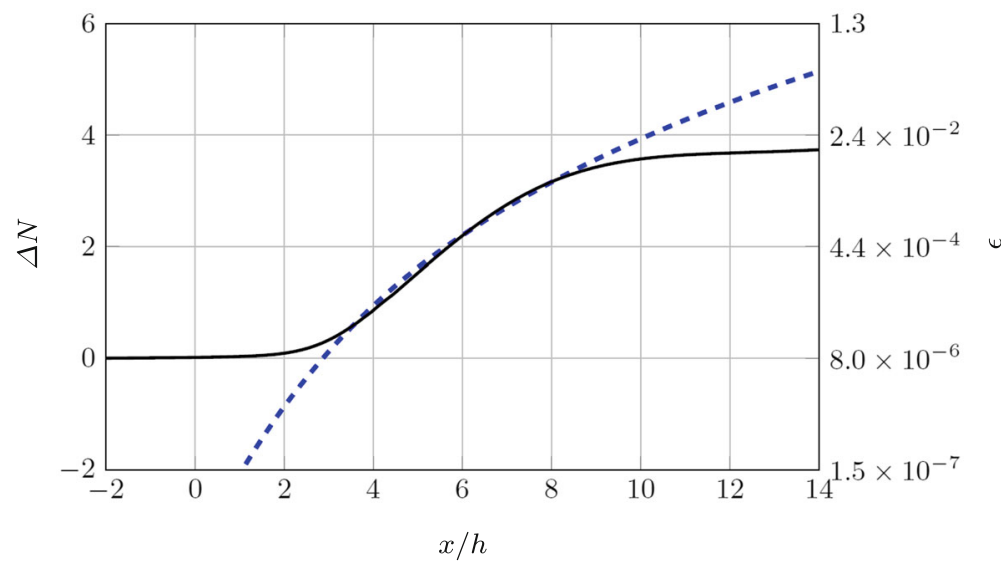

Fig. 5 Streamwise-velocity-perturbation amplitude from DNS (solid) and stability analysis (dashed). Left vertical axis, difference between $N(x / h)$, and $N(-2)$ computed from DNS, representative of the size of the perturbation velocity component; right vertical axis, $\epsilon$ values associated with each $\Delta N$, representative of the size of the streamwise perturbation kinetic energy 
reasonably neglected (the effect is small in the center of a wave packet and the formation is likely dependent on the numerical setup). The validity of this approximation is argued to be confirmed by the match of the $N$-factor curve with the amplitude evolution measured in the DNS as shown in Fig. 5.

Close to the micro-ramp, $\epsilon$ computed from DNS exhibits a plateau. This is ascribed to acoustic contamination, which sets an initial perturbation amplitude. At $x / h=3$, DNS perturbations start to grow exponentially at the same rate as the $N$-factor curve obtained from the results of the stability analysis. Exponential growth of disturbances is captured until $x / h \approx 8$. Further downstream, the growth rate of the DNS perturbations levels off with respect to the linear stability results, which signifies non-linear perturbation saturation.

Large-scale hairpins appear in the range $7 \leq x / h \leq 11$; see Figs. 3 and 6. In Fig. 3b we have identified these structures with the label "HP". In the aforementioned range, we additionally capture the onset of a secondary unsteady vortical structure, hereafter referred to as leg-buffer and labelled as "LB" in Fig. 3b,c. This structure was first reported in Ref. [11] and we initially capture it as a protrusion of the hairpin head, referred to as a duckling beak in Ref. [33]. In turn, the leg-buffer eventually induces a tertiary vortical structure, labelled as "LBIV" in Fig. 3b. We find a good topological match between the hairpin and legbuffer structures obtained from the results of DNS and tomo-PIV experiments in the range $x / h<30$; see Fig. 3b,c. Note that the tomo-PIV data was reconstructed from low-order modes after Proper Orthogonal Decomposition (POD). We notice differences regarding the streamwise inception location of the vortices and their wavelength. This is most probably induced by the different disturbance environment in the DNS versus the tomo-PIV experiments. Differences between DNS and most unstable stability eigenfunction could be attributed to non-linear effects.

Fig. 6 Streamwise-velocity perturbation from (a) the most unstable stability eigenfunction, (b) DNS,

(c) tomo-PIV experiments [11]. Perturbation with respect to (a) base and $(\mathbf{b}, \mathbf{c})$ mean flow

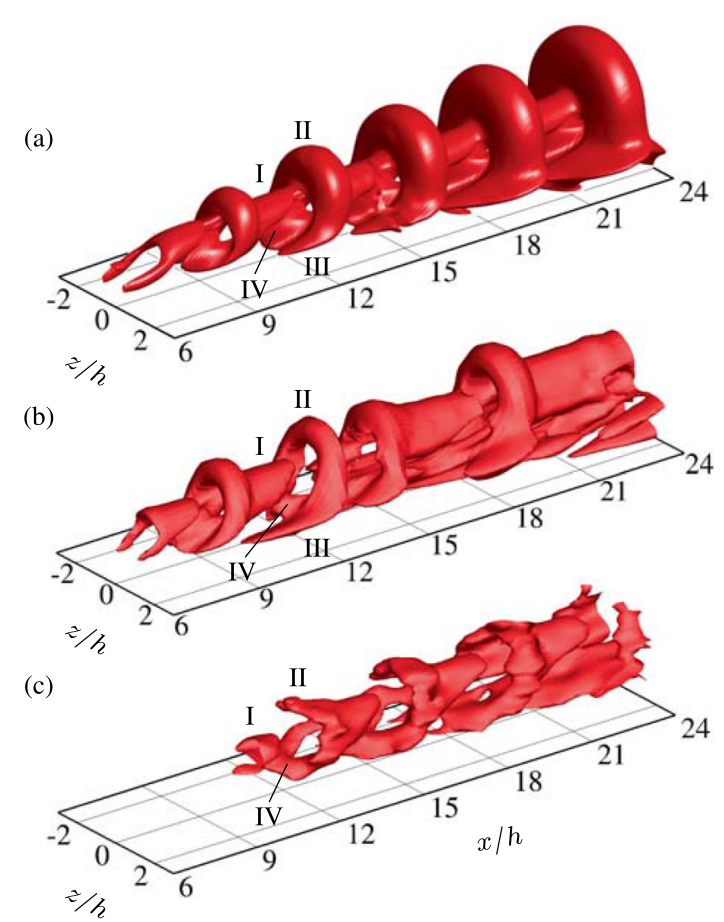


Along $11 \leq x / h \leq 30$, the head part of the hairpin vortices lifts up and straightens, as also pointed out in Ref. [33], the leg portion undergoes elongation while shifting downward, vortex-to-vortex distance is observed to gradually increase. In this range and for increasing $x / h$, the action of perturbations causes the leg-buffers to move forward and away from the centre plane, bifurcate into two branches featuring opposite streamwise vorticity and elongate. For $x / h>30$, the LBIVs and a branch of the original leg-buffer which separates and develops independently, significantly contribute to the broadening of the wake of the micro-ramp by introducing lateral disturbances.

The above described transitional perturbation structures enhance the momentum redistribution within the boundary layer. Figure 7a shows the cross-sectional contour of relevant vortex kinds at $x / h=21.5$, namely HPs and LBs. Sufficiently downstream of the microramp, and based on qualitative observations, this system of vortices generates a new dominant pattern of instantaneous wall-normal fluid motion. In Fig. 7a, we observe local upwash and downwash induced by HPs and LBs; the hairpin leg portions rotate in the same sense as the primary vortex pair and induce upwash close to the centre plane and lateral downwash. The LBs rotate in a sense opposite to the hairpin legs and thus act to enhance the hairpin-induced downwash near the wall while generating upwash aside. When moving downstream, we additionally capture weak activity of LBIVs, as illustrated in Fig. 7c. Far downstream of the micro-ramp, the instantaneous wall-normal velocity field features a spanwise-alternating pattern of upwash and downwash.

\subsection{Momentum excess evolution in the base flow and in the mean flow}

Along the micro-ramp and within its direct downstream vicinity, the action of the primary vortices highly distorts the structure of the boundary layer. High-momentum fluid from intermediate portions of the boundary layer penetrates into the low-momentum region under the action of downwash. In parallel, the upwash moves low momentum fluid from the lower

Fig. 7 Instantaneous $\lambda_{2}$ isosurface from DNS (b) with $y-z$ planes of wall-normal velocity at $(\mathbf{a}, \mathbf{b}) x / h=21.5$; (b,c) $x / h=42.2$. Iso-contour of $\lambda_{2}(\mathbf{a}, \mathbf{c})$ (black solid line)

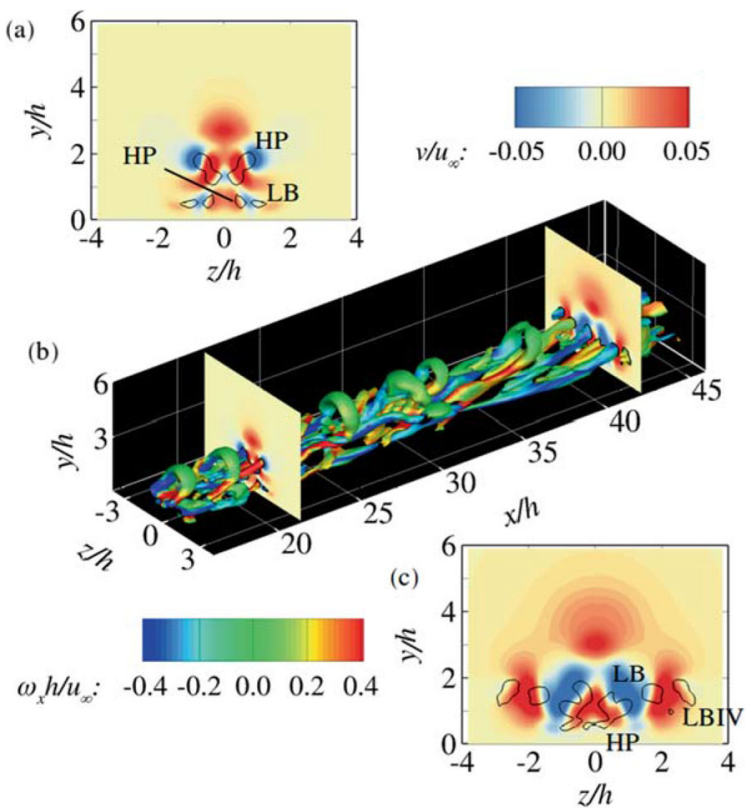


portion of the boundary layer upwards. Consequently, a momentum excess region, in which the streamwise momentum is larger than in the unperturbed boundary layer $\left(\langle u\rangle>u_{\mathrm{un}}\right)$, is initially generated aside the centre plane and near the surface, and a central momentum deficit region, in which the streamwise momentum is lower than in the unperturbed boundary layer $\left(\langle u\rangle<u_{\mathrm{un}}\right)$, originates around the micro-ramp tip.

The regions of momentum excess and deficit are characterised via the streamwisevelocity-streak field, the difference between the local streamwise velocity and the unperturbed boundary layer: $u^{\text {str }}=u-u_{\text {un }}$. Figure 8 depicts contours of the base and the mean streamwise-velocity-streak field for two planes at $x / h=20$ and 80 . Close to the microramp, in absence of significant perturbation activity, the regions of momentum excess and deficit in the base and in the mean flow match qualitatively. However, we observe significant differences between mean and base flow further downstream, which is the footprint of the non-linear finite-amplitude perturbations developing.

When moving downstream in the base flow, the base-flow streak field seems to diffuse, that is, the excess and deficit regions grow in size while their amplitude decreases. Far enough downstream, the primary vortex pair becomes incapable of maintaining the entrainment of high-momentum fluid close to the wall. This is assumed to be mainly a consequence of the initial strong lift-up of the primary vortices, together with the decreasing lateral downwash in the streamwise direction. Far downstream of the micro-ramp, the nearwall fluid layers evolve back towards a configuration resembling the unperturbed boundary layer and, as a consequence, feature overall smaller values of $u_{\mathrm{s}}^{\text {str }}$ than in the near-ramp field (Fig. 8a,c). These results are conform with the significant decrease of the wall shear in the base flow for $x / h>10$ illustrated in Fig. 4a. When considering the mean flow instead, we observe that sufficiently downstream of the micro-ramp, the lower portion of the boundary layer aside the centre plane becomes progressively fuller. Clearly, perturbations effectively entrain high-momentum fluid near the wall.

The wall-normal velocity field in the base flow undergoes a strong decrease in magnitude along the $x$-direction; as illustrated in Fig. 9a,c. In the mean flow field, however, up- and downwash regions maintain their strength and expand upwards and sidewards; see
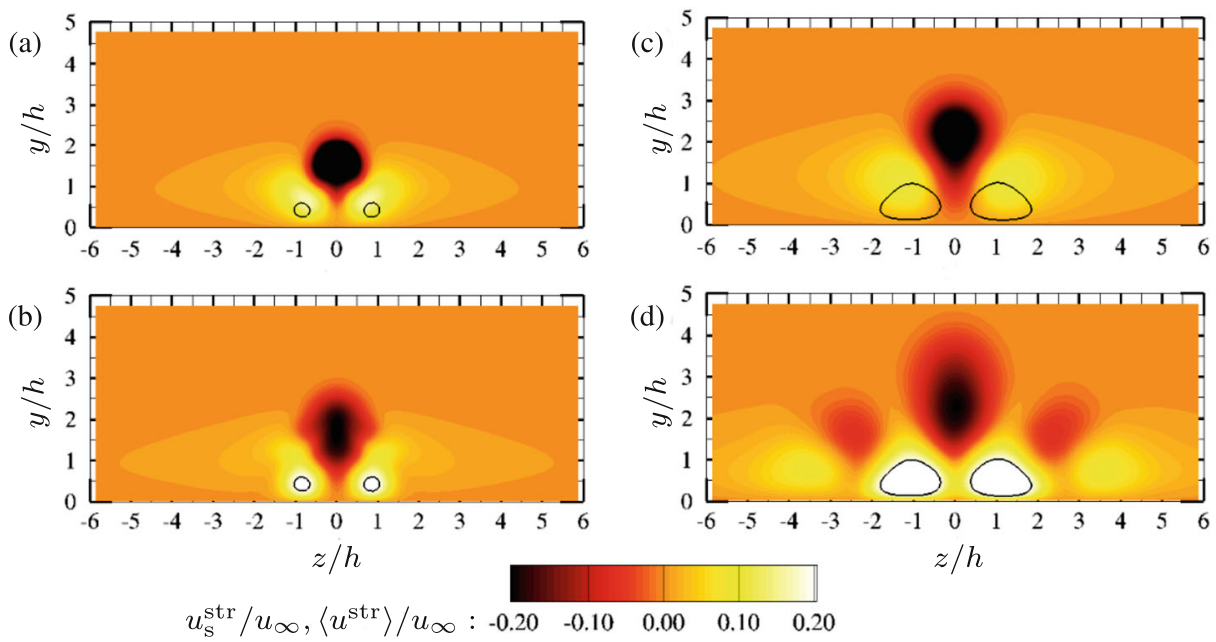

Fig. 8 Streamwise-velocity streaks in the base flow (a,c) and mean flow (b,d) at $x / h=20(\mathbf{a}, \mathbf{b})$ and 80 (c,d). Iso-contours of $\left\langle u^{\text {str }}\right\rangle / u_{\infty}=0.2$ (black line) 

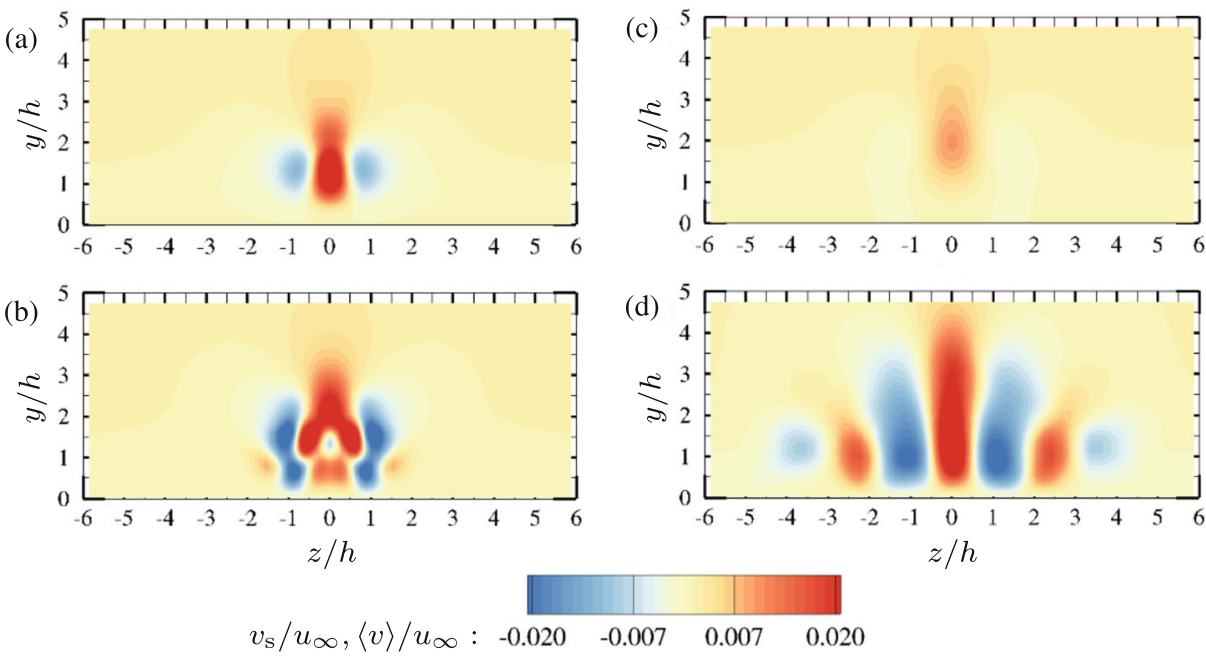

Fig. 9 Wall-normal velocity in the base flow (a,c) and mean flow (b,d) at $x / h=20(\mathbf{a}, \mathbf{b})$ and $80(\mathbf{c}, \mathbf{d})$

Fig. 9b,d. Hairpin vortices and secondary vortices introduce the spanwise-alternating pattern of upwash and downwash in the instantaneous flow field (Fig. 7), which is captured in the mean flow as well. The perturbations enhance the redistribution of momentum and gradually modulate the mean flow boundary layer in the spanwise direction. This explains that already at $x / h=20$, the cores of the momentum excess regions in the mean flow become relatively stronger than in the base flow (Fig. 8a,b). Further downstream, this trend is more pronounced: momentum excess in the mean flow is dominant near the wall and much stronger than in the base flow (Fig. 8c,d). This is consistent with the significant increase of the wall shear in the mean flow for large $x / h$. The largest values of $\left\langle u^{\text {str }}\right\rangle$ are observed at $|z / h| \approx 1$, corresponding to the spanwise location of downwash in the mean flow (Figs. 8d and 9d). We observe further regions of added momentum aside the central ones not present in the base flow; it is suggested that these are generated solely due to the action of secondary vortices.

The aforementioned qualitative observations are quantified by evaluating the streamwise evolution of the momentum excess contained in the boundary layer. For this purpose, Fig. 10 shows the evolution of

$$
\begin{aligned}
& E_{\mathrm{B}}(x)=\int_{A^{\mathrm{E}}} \frac{\rho u_{\mathrm{s}}^{\mathrm{str}}}{\rho_{\infty} u_{\infty}} \mathrm{d}\left(\frac{A}{h^{2}}\right), \\
& E_{\mathrm{M}}(x)=\int_{A^{\mathrm{E}}} \frac{\left\langle\rho u^{\mathrm{str}}\right\rangle}{\rho_{\infty} u_{\infty}} \mathrm{d}\left(\frac{A}{h^{2}}\right),
\end{aligned}
$$

where $E_{\mathrm{B}}$ and $E_{\mathrm{M}}$ represent the momentum integrated over the $y-z$ planes $A^{\mathrm{E}}$ where $u_{\mathrm{s}}^{\text {str }}$ and $\left\langle u^{\text {str }}\right\rangle$ are positive and $\rho_{\infty}$ denotes the free-stream density. In the base flow, the generation of momentum excess is not limited to the micro-ramp location, but the amount of momentum excess in the boundary layer eventually stabilises in the downstream direction. In the mean flow, from $x / h \approx 20$ onward, the amount of added momentum becomes increasingly larger than in the base flow, reaching a significantly higher value at $x / h=90$. 
Fig. 10 Evolution of momentum excess in the base flow $\left(E_{\mathrm{B}}\right.$, solid line) and mean flow ( $E_{\mathrm{M}}$, dashed line)

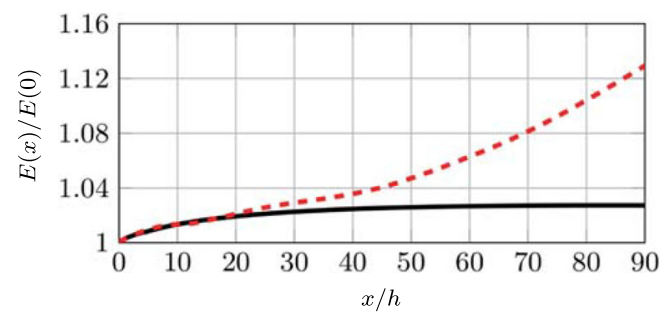

\subsection{Transport of momentum streaks}

In an attempt to quantitatively assess the aforementioned differences in momentum excess generation, we derive streamwise-momentum-streak transport equations for the base flow and mean flow. We consider incompressible flow conditions and constant viscosity, which is a reasonable approximation for $M_{\infty}<0.3$. In the unperturbed boundary layer, the spanwise velocity is zero $\left(w_{\text {un }}=0\right)$, and the streamwise and wall-normal velocity components are independent of the spanwise coordinate $\left(\partial u_{\mathrm{un}} / \partial z=0\right.$ and $\left.\partial v_{\mathrm{un}} / \partial z=0\right)$.

Since we are interested in mathematically characterising the behaviour of the momentum excess, we propose to further decompose the flow field. Letting $q$ be any flow variable, the decomposition for the base flow analysis reads:

$$
q_{\mathrm{s}}=q_{\mathrm{un}}+q_{\mathrm{s}}^{\mathrm{str}},
$$

i.e., the base flow field is expressed as the sum of the unperturbed boundary layer, $q_{\text {un }}$, and the streak field, $q_{\mathrm{s}}^{\text {str }}$. For the mean flow analysis, we use the decomposition

$$
q=q_{\mathrm{un}}+\left\langle q^{\mathrm{str}}\right\rangle+q^{\prime \prime},
$$

where $q^{\prime \prime}$ expresses an instantaneous fluctuation measured with respect to the mean-flow velocity field.

By introducing Definition (9) into the steady streamwise-momentum conservation equation, we obtain an equation describing the transport of momentum streaks in a steady flow field:

$$
\underbrace{\rho\left(\boldsymbol{v}_{\text {un }} \cdot \nabla\right) u_{\mathrm{s}}^{\text {str }}}_{\text {streak advection }}=\underbrace{-\frac{\partial p_{\mathrm{s}}^{\mathrm{str}}}{\partial x}}_{\text {pressure }}+\underbrace{\mu \nabla^{2} u_{\mathrm{s}}^{\mathrm{str}}}_{\text {diffusion }}+\underbrace{\nabla \cdot \overbrace{\left(-\rho u_{\mathrm{s}} \boldsymbol{v}_{\mathrm{s}}^{\mathrm{str}}\right)}^{\text {streak stress }}}_{\text {streak }- \text { shear}- \text { correlation }} .
$$

Besides the expected advection, pressure and viscous diffusion terms, one additionally obtains an extra term, which we refer to as streak-shear-correlation and interpret as the divergence of an apparent streak stress, $-\rho u_{\mathrm{s}} \boldsymbol{v}_{\mathrm{s}}^{\mathrm{str}}$.

With Expression (10) and the assumption of a converged statistically steady flow with $\partial\langle\rangle / \partial t=0$, we obtain the mean-flow streaks budget:

$$
\underbrace{\rho\left(\boldsymbol{v}_{\text {un }} \cdot \nabla\right)\left\langle u^{\text {str }}\right\rangle}_{\text {streak advection }}=\underbrace{-\frac{\partial\left\langle p^{\text {str }}\right\rangle}{\partial x}}_{\text {pressure }}+\underbrace{\mu \nabla^{2}\left\langle u^{\text {str }}\right\rangle}_{\text {diffusion }}+\underbrace{\nabla \cdot \overbrace{\left(-\rho\langle u\rangle\left\langle\boldsymbol{v}^{\text {str }}\right\rangle\right)}^{\text {streak stress }}}_{\text {streak-shear-correlation }}+\nabla \cdot \overbrace{\left(-\rho\left\langle u^{\prime \prime} \boldsymbol{v}^{\prime \prime}\right\rangle\right.}^{\text {Reynolds stress }}),
$$

which describes the transport of momentum streaks in a mean flow field. Equation 12 shows a structure similar to Eq. 11, but adds the Reynolds-stress term. Congruence between terms in Eqs. 11 and 12 allows us to compare their relative contribution to the generation 
and destruction of streamwise-momentum streaks in the base and mean configurations of the micro-ramp wake. Differences can be attributed to the non-linear effect of perturbation development.

The streamwise evolution of each of the terms in Eqs. 11 and 12 integrated over the regions of added momentum in the base and mean flow are presented in Fig. 11. Each integral term characterises the relative contribution of each of the different streak transport mechanisms to the local generation of momentum excess, which we associate with the integral contribution of the streak advection term. In Fig. 12, we reproduce Fig. 11 zooming in the range $-5 \leq x / h \leq 10$.

The results in Fig. 12 indicate that, in the range $-5 \leq x / h \leq 10$, streak stresses (blue circles) act so as to generate momentum excess; the associated curve departs from the horizontal axis at $x / h \approx-2$ and lies in the positive half-plane. Diffusion (magenta diamonds) plays an opposite role, it is of a destructive nature. Reynolds stresses (red solid line) do not play a role in the base flow; in the mean flow, in absence of significant perturbation activity, the contribution of Reynolds stresses to the generation of positive momentum streaks is virtually zero close to the micro-ramp. This is conform with the small perturbation amplitudes. The pressure term (green crosses) first contributes positively and, for $x / h>-0.75$, it contributes negatively.

The behaviour of the pressure term in Fig. 12 can be explained upon inspection of the flow field: upstream of the micro-ramp tip, $\partial p / \partial x$ initially becomes negative at the location of the momentum excess regions due to the formation of the vortex cores. Hence, for increasing $x / h$, the pressure term in Eqs. 11 and 12 initially becomes positive and peaks at $x / h \approx-1.7$. From $x / h \approx-1.7$ onward, the contribution of the pressure in streak generation first decreases and thereafter $(x / h>-0.75)$ becomes destructive due to the fact that pressure recovery in streamwise direction yields $\partial p / \partial x>0$ in both the base and in the mean flow fields. Thus, our results indicate that pressure plays a significant role in the

(a)

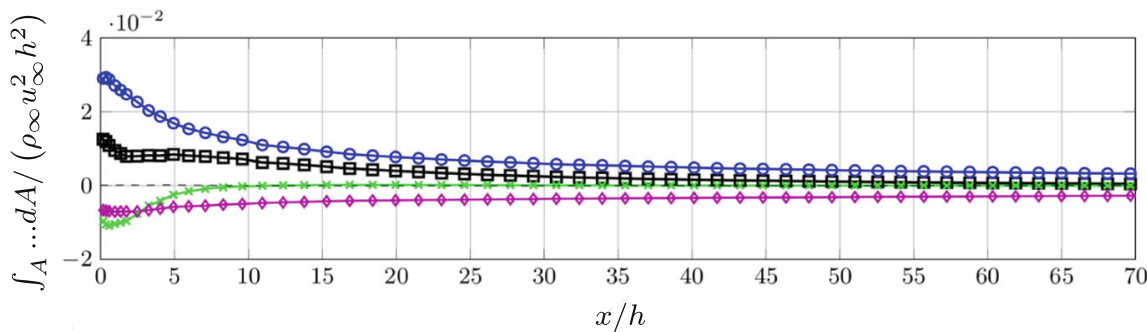

(b)

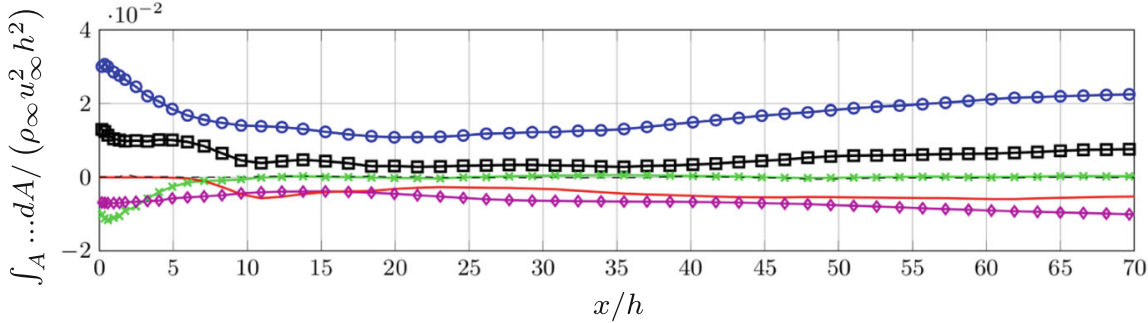

Fig. 11 Streamwise evolution of the terms of (a) (11) and (b) (12) integrated over the regions of momentum excess in (a) the base flow and (b) the mean flow. Streak advection (black squares), streak-shear-correlation (blue circles), pressure (green crosses), diffusion (magenta diamonds), Reynolds stresses (red solid line), local sum of terms (black dashed line) 


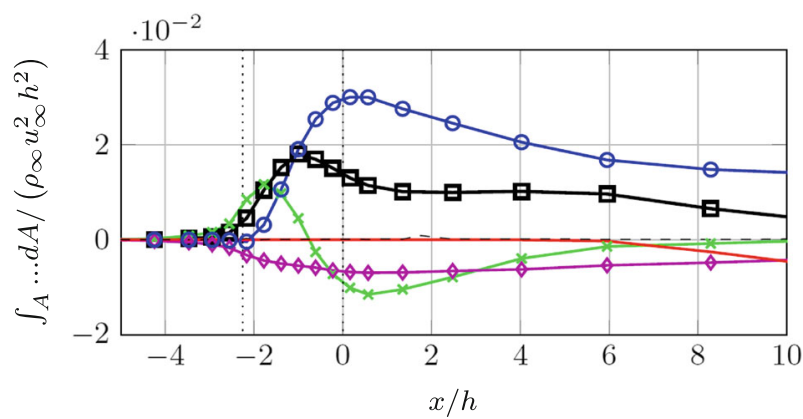

Fig. 12 Streamwise evolution of the terms of Eq. 12 integrated over the regions of momentum excess in the mean flow. Streak advection (black squares), streak-shear-correlation (blue circles), pressure (green crosses), diffusion (magenta diamonds), Reynolds stresses (red solid line), local sum of terms (black dashed line), streamwise position of the micro-ramp's leading edge and tip $(x / h=-2.25,0)$ (vertical dotted lines)

initial generation of momentum excess at and near the micro-ramp location. As soon as the pressure introduces gradients of $\rho u^{\text {str }}$, diffusion starts playing a role as well. The largest productive contribution comes from streak stresses. The associated curve in Fig. 12 first becomes non-zero at $x / h=-2.1$, near the position of the micro-ramp's leading edge, and peaks at $x / h \approx 0$, the position of the micro-ramp's tip. Along this streamwise range, the primary vortices are induced and produce an initial strong downwash.

Through visual inspection, we first detect regions of positive momentum streaks in the flow field upstream of the micro-ramp at $x / h \approx-3$. This coincides with the streamwise station at which the pressure curve in Fig. 12 starts to reach higher absolute values than that of diffusion. Although the contribution of pressure eventually decreases, the rapid streamwise growth of streak-stress activity maintains monotonically increasing momentum streaks generation until it attains a maximum at $x / h=-1$. From this point onward, even though the generation of positive streamwise-momentum streaks decreases, it remains significant over a short distance downstream of the micro-ramp. This partially disagrees with conclusions drawn in Ref. [17], inasmuch as it is claimed that the mechanism of the micro-ramp is position alternation of high- and low-momentum fluid triggered by the primary vortices at the micro-ramp rather than downstream of it.

Base flow results in Fig. 11a indicate that for $x / h>10$, the pressure does not add a significant contribution to the generation of positive momentum streaks and that the contribution of diffusion is maintained negative and rather constant. Streak stresses have been shown to play the central role in the generation of momentum excess at the micro-ramp and at its immediate downstream vicinity. Current results show that the curve of streak stresses decreases monotonically downstream of the micro-ramp in the base flow. Gradually, their contribution approaches that of diffusion in absolute value and eventually, streak stresses practically balance the effect of diffusion. As a consequence, for $x / h>50$, the streak advection curve (black squares) approaches zero from above. Thus, at these downstream locations, the momentum excess generated upstream is conserved; it is essentially transported downstream without further significant addition or subtraction.

For the mean flow field the Reynolds stress additionally contributes to the evolution of momentum streaks. As mentioned previously, the streamwise evolution of the curves in Fig. 11a and $\mathrm{b}$ resemble each other close to the micro-ramp. For $x / h>5$, however, as perturbations grow gradually, the Reynolds stress term noticeably departs from the horizontal axis, becomes negative, and so it yields a destructive contribution (Fig. 11b). Nonetheless, 
the effect of disturbance activity in the transport of momentum streaks is not completely characterised by the Reynolds stress term alone. The mean flow distortion also manifests itself through the adaptation of the streak stresses and diffusion. At $x / h=11.5$, the curve for the Reynolds stresses reaches a local minimum: the varicose instability has given rise to the first generation of hairpin vortices at that point, see Fig. 3. As a consequence of the significant increase in Reynolds stress activity, the generation of momentum excess momentarily becomes smaller than in the base flow around this streamwise position. The contribution of the Reynolds stress term remains destructive throughout the entire domain and, like diffusion, increases in absolute value for large $x / h$.

The monotonic decrease of the streak-stress curve along $x / h$ in the base flow highlights that the laminar primary vortices gradually lose their capability to generate momentum excess. In the mean flow, however, the curve of the streak stresses -which adds the largest productive contribution- reaches a global minimum at $x / h=21$. Perturbation motion takes over the role of the primary vortex pair in redistributing momentum within the boundary layer. The streak stress term grows enough to counter-act the negative effect of the viscous diffusion and the Reynolds stresses, and maintains the generation of momentum excess increasingly positive for large $x / h$.

\section{Conclusions}

We studied the wake dynamics of a micro-ramp vortex generator immersed in a quasiincompressible $\left(M_{\infty}=0.2\right)$ boundary layer at supercritical roughness Reynolds number $\left(R e_{h h}=463\right)$ conditions. We performed Direct Numerical Simulations (DNS) of the laminar base flow and the linear and non-linear transitional flow, from which we obtained the time-averaged mean flow. The DNS results were compared with the results of independent tomographic Particle Image Velocimetry (tomo-PIV) experiments and BiLocal stability analysis on the computed base flow. The DNS results for the instantaneous flow are in good agreement with tomo-PIV experiments, and we obtained matching linear disturbance growth rates when comparing the results of DNS and stability analysis.

Upon computation of the unstable base flow solution, we captured the structure of the primary vortices in absence of transitional perturbation evolution. We observed that the laminar primary vortices can only produce a significant entrainment of high-momentum fluid towards the wall at the micro-ramp location and over a very short distance downstream from it. In the base flow, the lower portion of the boundary layer aside the centre plane undergoes a recovery in the streamwise direction. This was hypothesised to be a consequence of the rapid upward shift of the primary vortices due to their self-induced wall-normal velocity and the steep reduction of the lateral vortex-induced downwash in the streamwise direction due to the decay of vortex intensity.

In the instantaneous transitional flow field, the primary vortices break up into a set of large-scale hairpin vortices induced at the detached shear layer, which we identified as the main instantaneous flow structures. We also captured secondary vortices, referred to as leg-buffers, initially developing in between hairpin vortices. Secondary vortices in the transitional flow field play an important role in expanding the wake of the micro-ramp in the spanwise direction since their action introduces perturbation contamination away from the centre plane. Overall, we found a good topological match between the instantaneous transitional vortical structures obtained from the DNS and a low-order POD reconstruction based on tomo-PIV data. Good agreement was found as well for the streamwise-velocity perturbation structure from the results of DNS and tomo-PIV with the most unstable stability 
eigenfunction. Exponential growth of disturbances in agreement with linear stability theory was captured until eight micro-ramp heights downstream from the micro-ramp.

Together, hairpin vortices and secondary vortices are more effective than the base flow primary vortices in enhancing the momentum redistribution within the boundary layer. Perturbation activity produces a sustained entrainment of high-momentum fluid towards the surface near the centre plane. As a consequence, the mean flow boundary layer in this region becomes fuller than the base flow boundary layer. Whereas upwash and downwash induced by the primary vortex pair in the base flow decrease their strength far downstream of the micro-ramp, the mean wall-normal velocity field displays a spanwise-alternating pattern of upwash and downwash of larger magnitude, which modulates the mean flow boundary layer in the spanwise direction.

We evaluated the streamwise-momentum-streak transport equation in order to quantify the contributions of the different streak transport mechanisms to the generation of momentum excess. The mechanism referred to as the streak-shear correlation is the dominant production term in both the base flow and mean flow. In the base flow, the contribution of the streak stresses gradually decays in the streamwise direction and ultimately essentially counter-acts the negative effect of viscous diffusion, whereas in the mean flow, it is increasingly positive far downstream of the micro-ramp and maintains the generation of momentum excess.

For the flow conditions considered in this article, we conclude that the micro-ramp does not operate as the classical vortex generator which relies on the action of downstreamevolving vortex filaments. We have shown that the transitional perturbations increase the near-wall momentum in the mean flow more efficiently than the primary vortices in the laminar base flow.

Acknowledgements This work was carried out on the Dutch national e-infrastructure with the support of SURF Cooperative.

\section{Compliance with Ethical Standards}

Conflict of interests The authors declare that they have no conflict of interest.

Open Access This article is distributed under the terms of the Creative Commons Attribution 4.0 International License (http://creativecommons.org/licenses/by/4.0/), which permits unrestricted use, distribution, and reproduction in any medium, provided you give appropriate credit to the original author(s) and the source, provide a link to the Creative Commons license, and indicate if changes were made.

\section{References}

1. Tani, I.: A survey of the effect of small protuberances on boundary-layer flows. AIAA J. 11, 169-196 (1969)

2. Kachanov, Y.S.: Physical mechanisms of laminar-boundary-layer transition. Annu. Rev. Fluid Mech. 26, 411-482 (1994)

3. Ergin, F.G., White, E.B.: Unsteady and transitional flows behind roughness elements. AIAA J. 44, 25042513 (2006)

4. Klebanoff, P.S., Tidstrom, K.D.: Mechanism by which a two-dimensional roughness element induces boundary-layer transition. Phys. Fluids 15, 1173-1188 (1972)

5. Cossu, C., Brandt, L.: On Tollmien-Schlichting-like waves in streaky boundary layers. Eur. J. Fluid Mech. (B/Fluids) 23, 815-833 (2004)

6. Schneider, S.P.: Effects of roughness on hypersonic boundary-layer transition. J. Spacecr. Rockets $\mathbf{4 5}$, 193-209 (2008) 
7. Redford, J.A., Sandham, N.D., Roberts, G.T.: Compressibility effects on boundary-layer transition induced by an isolated roughness element. AIAA J. 48, 2818-2830 (2010)

8. Acarlar, M.S., Smith, C.R.: A study of hairpin vortices in a laminar boundary layer. Part 1. Hairpin vortices generated by a hemisphere protuberance. J. Fluid Mech. 175, 1-41 (1987)

9. Klebanoff, P.S., Cleveland, W.G., Tidstrom, K.D.: On the evolution of a turbulent boundary layer induced by a three-dimensional roughness element. J. Fluid Mech. 237, 101-187 (1992)

10. Loiseau, J.-C., Robinet, J.-C., Cherubini, S., Leriche, E.: Investigation of the roughness-induced transition:, global stability analyses and direct numerical simulations. J. Fluid Mech. 760, 175-211 (2014)

11. Ye, Q., Schrijer, F.F.J., Scarano, F.: On Reynolds number dependence of micro-ramp-induced transition. J. Fluid Mech. 837, 597-626 (2018)

12. Anderson, B.H., Tinapple, H., Surber, L.: Optimal control of shock wave turbulent boundary layer interactions using micro-array actuation. AIAA Paper 2006-3197 (2006)

13. Babinsky, H., Li, Y., Pitt Ford, C.W.: Microramp control of supersonic oblique shock-wave/boundarylayer interactions. AIAA J. 47, 668-674 (2009)

14. Giepman, R.H.M., Schrijer, F.F.J., van Oudheusden, B.W.: Flow control of an oblique shock wave reflection with micro-ramp vortex generators: Effect of location and size. Phys. Fluids 26, 1-16 (2014)

15. Lin, J.C.: Review of research on low-profile vortex generators to control boundary-layer separation. Prog. Aerosp. Sci. 38, 389-420 (2002)

16. Tirtey, S.C., Chazot, O., Walpot, L.: Characterization of hypersonic roughness-induced boundary-layer flow. Exp. Fluids 50, 407-418 (2011)

17. Wang, X., Yan, Y., Sun, Z., Liu, C.: LES investigation into the generation of momentum deficits in the supersonic wake of a micro-ramp. J. Mech. Sci. Technol. 28, 1327-1337 (2013)

18. Li, Q., Liu, C.: Implicit LES for supersonic microramp vortex generator: New discoveries and new mechanisms. Model. Simul. Eng. 2011, 15 (2011)

19. Bo, W., Weidong, L., Yuxin, Z., Xiaoqiang, F., Chao, W.: Experimental investigation of the micro-ramp based shock wave and turbulent boundary layer interaction control. Phys. Fluids 24, 055110 (2012)

20. Blinde, P.L., Humble, R.A., van Oudheusden, B.W., Scarano, F.: Effects of micro-ramps on a shock wave/turbulent boundary layer interaction. Shock Waves 19, 507-520 (2009)

21. Hickel, S., Egerer, C.P., Larsson, J.: Subgrid-scale modeling for implicit large eddy simulation of compressible flows and shock-turbulence interaction. Phys. Fluids 26, 106-101 (2014)

22. Örley, F., Hickel, S., Pasquariello, V., Adams, N.: Cut-element based immersed boundary method for moving geometries in compressible liquid flows with cavitation. J. Comp. Phys. 283, 1-22 (2015)

23. Liu, X.-D., Osher, S., Chan, T.: Weighted essentially non-oscillatory schemes. J. Comp. Phys. 115, 200-212 (1994)

24. Toro, E.F., Spruce, M., Speares, W.: Restoration of the contact surface in the HLL-Riemann solver. Shock Waves 4, 25-34 (1994)

25. Åkervik, E., Brandt, L., Henningson, D.S., Hœpffner, J., Marxen, O., Schlatter, P.: Steady solutions of the Navier-Stokes equations by selective frequency damping. Phys. Fluids 18, 357-397 (2006)

26. Casacuberta, J., Groot, K.J., Tol, H.J., Hickel, S.: Effectivity and efficiency of selective frequency damping for the computation of unstable steady-state solutions. J. Comp. Phys. 375, 481-497 (2018)

27. Huerre, P., Monkewitz, P.A.: Absolute and convective instabilities in open shear layers. J. Fluid Mech. 159, 151-168 (1985)

28. Huerre, P., Monkewitz, P.A.: Local and global instabilities in spatially developing flows. Annu. Rev. Fluid Mech. 22(1), 473-537 (1990)

29. Theofilis, V.: Advances in global linear instability analysis of nonparallel and three-dimensional flows. Progress Aerosp. Sci. 39(4), 249-315 (2003)

30. Bridges, T.J., Morris, P.J.: Differential eigenvalue problems in which the parameter appears nonlinearly. J. Comput. Phys. 55(3), 437-460 (1984)

31. Canuto, C., Hussaini, M.Y., Quarteroni, A., Zang, T.A.: Spectral Methods: Fundamentals in Single Domains. Springer (2006)

32. Anderson, E., Bai, Z., Bischof, C., Blackford, L.S., Demmel, J., Dongarra, J.J., Du Croz, J., Hammarling, S., Greenbaum, A., McKenney, A., Sorensen, D.: LAPACK Users' guide. Society for Industrial and Applied Mathematics (1999)

33. Groot, K.J., Ye, Q., van Oudheusden, B.W., Zhang, Y., Pinna, F.: BiGlobal stability analysis of a microramp wake using PIV base flows. 46th AIAA Fluid Dynamics Conference (2016)

34. Groot, K.J., Serpieri, J., Pinna, F., Kotsonis, M.: Secondary crossflow instability through global analysis of measured base flows. J. Fluid Mech. 846, 605-653 (2018)

Publisher's Note Springer Nature remains neutral with regard to jurisdictional claims in published maps and institutional affiliations. 